\title{
Subsurface Confinement: Evidence from Submariners of the Benefits of Mindfulness
}

\author{
Charlotte Aufauvre-Poupon ${ }^{1} \cdot$ Charles Martin-Krumm ${ }^{2,3}$. Anais Duffaud ${ }^{4,5} \cdot$ Adrien Lafontaine $^{5,6} \cdot$ Lionel Gibert $^{4,7}$. \\ Fabien Roynard ${ }^{4}$. Christophe Rouquet ${ }^{4}$. Jean-Baptiste Bouillon-Minois ${ }^{8,9} \cdot$ Frédéric Dutheil $^{8,10} \cdot$ Frédéric Canini $^{4,5}$. \\ Julien Pontis ${ }^{11}$ - François Leclerq ${ }^{11}$. Alexandre Vannier ${ }^{11}$ - Marion Trousselard ${ }^{2,4} \mathbb{C}$
}

Accepted: 21 June 2021 / Published online: 9 July 2021

(c) The Author(s), under exclusive licence to Springer Science+Business Media, LLC, part of Springer Nature 2021

\begin{abstract}
Objectives The subsurface ballistic missile nuclear submarine (SSBN) is an extreme professional environment in which personnel are both isolated and confined during patrols, which can last longer than 2 months. This environment is known to degrade submariners' mood and cognition.

Methods This exploratory, empirical study followed a cohort of 24 volunteer submariners. Dispositional mindfulness was assessed with the Freiburg Mindfulness Inventory, in order to identify two groups (mindful and non-mindful) and compare change in emotional state, interoception, and health behaviors during the patrol.

Results Overall, psychological health deteriorated during the patrol. However, mindful submariners demonstrated better psychological adaptation and interoception than the non-mindful group. This was associated with better subjective health behaviors (sleeping and eating).

Conclusions Dispositional mindfulness appears to protect against the negative effects of long-term containment in a professional environment, such as a submarine patrol. Our work highlights that mindfulness may help individuals to cope with stress in such situations. Developing mindfulness could also be an important preventive healthcare measure during quarantine imposed by the outbreak of a serious infectious disease.
\end{abstract}

Keywords Containment $\cdot$ Mindfulness $\cdot$ Submarine $\cdot$ Psychological health $\cdot$ Health behaviors

Long-term space missions require a good understanding of human adaptation to artificial environments, known as

Marion Trousselard

marion.trousselard@gmail.com

1 École Camondo, 266 Boulevard Raspail, 75014 Paris, France

2 APEMAC/EPSAM, EA 4360, Ile du Saulcy, BP 30309 , 57006 Metz, Cedex 1, France

3 École de Psychologues Praticiens, Institut Catholique de Paris (Catholic Institute of Paris), VCR/ICP EA 7403-23, Rue du Montparnasse, 75006 Paris, France

4 French Armed Forces Biomedical Research Institute, BP73, 91223 Brétigny-sur-Orge, Cedex, France

5 Réseau $\mathrm{ABC}$ des psychotraumas; http://www.abcpsychotraumas.fr/, Montpellier, France

6 French Military Health Service Academy, 1 Place Alphonse Laveran, 75230 Paris, Cedex 05, France isolated and confined environments (ICE), and/or extreme and unusual environments (EUE). The literature shows

7 Centre Hospitalier Universitaire Paul Brousse, Unité de Recherche PsychiatrieComorbidités-Addictions, PSYCOMADD, Villejuif, France

8 Université Clermont Auvergne, CNRS, LaPSCo, Physiological and Psychosocial Stress, 34 avenue Carnot, 63037 Clermont-Ferrand, France

9 University Hospital of Clermont-Ferrand, CHU Clermont-Ferrand, Emergency Medicine, F-63000 Clermont-Ferrand, France

10 University Hospital of Clermont-Ferrand, CHU Clermont-Ferrand, Occupational and Environmental Medicine, WittyFit, F, 63000 Clermont-Ferrand, France

11 French Submarines Forces Health Service, Brest, France 
that the constraints inherent in ICE and EUE can upset the balance between the demands of the environment, and the resources mobilized by individuals, leading to changes in the individual-environment relationship and stress disorders (Vanhove et al., 2015). As Rivolier (1992, p2) indicated, with respect to the extreme nature of certain situations, "the individual is placed in circumstances with intense emotional potential or requiring an adaptive response, an adjustment, which he experiences as beyond his means." Therefore, it is relevant to ask, what individual characteristics predict successful adaptation and performance in an ICE/EUE, especially long-term missions. The literature highlights, in conjunction with changes in environmental sensory stimuli, emotional and mood disorders (Brasher et al., 2010; Palinkas et al., 2007), attentional and cognitive difficulties (Palinkas et al., 1997), and social problems (Palinkas et al., 2004) that underlie maladaptive stress responses (Vanhove et al., 2015). These disorders appear during the mission, in individuals who have no apparent prior health concerns. These data suggest that sensory immersion in such environments may have deleterious effects on physical, mental, and cognitive health, through mechanisms that affect sensory integration.

The subsurface ballistic missile nuclear submarine (SSBN) is an unusual, confined, isolated, and sometimes extreme context. Along with Antarctic and space bases, it is an appropriate environment for studying human adaptation to a long-term mission. A patrol lasts several weeks and, during this time, submariners are subject to numerous constraints, often arising from their environment. In the case of the SSBN, besides the constrained living environment, they live alongside a nuclear weapon and the fear of potentially fatal damage. They must also maintain a watch-keeping cycle that disrupts the circadian rhythm, and cope with social confinement, along with a monotonous environment. These constraints have been found to affect emotion (Eid et al., 2001) and stress regulation (Brasher et al., 2010), but not sleep (Trousselard et al., 2015). This professional setting immerses individuals in a very different sensory environment. There can be a lack of sensory stimulation, a lack of variation in stimulation, or an overstimulation of a sensory modality. While the abnormal regulation and integration of sensory, perceptual, and attentional processes is well-known to be actively involved in the adaptive response of subjects whose cognitive functioning is affected by physical environmental constraints (Levit-Binnun \& Golland, 2012), these processes remain little-explored in healthy individuals. On the one hand, the adaptive ability to respond appropriately in a constantly changing environment involves a fine-tuned interplay between inside and outside, the brain and the body (Craig, 2009). Through the body, the brain receives information about the state of the external world (exteroception) and the body's physiological state (interoception). On the other hand, health benefits are associated with a natural environment (Sarris et al., 2019). In particular, naturalistic stimuli have been found to have restorative effects, compared to artificial stimuli (Gould van Praag et al., 2017).

Overall, the literature suggests that the operational constraints of the SSBN challenge the adaptability of submariners, who are immersed in an artificial, stressful environment. Meeting this challenge requires efficient interoception, which is the individual's ability to pay attention to information from the body and to notice subtle changes that are consistent with the available environmental information (Mehling et al., 2009). Interoception is a complex concept that includes objective processes of neural coding, transduction, and the central representation of internal stimuli (Salvato et al., 2020). It engages both afferent and efferent mechanisms via three steps: perception, attention, and awareness (Schulz \& Vogele, 2015). Although the first two steps are considered to be preconscious (Mehling et al., 2009), research has found that perceptions do not need to reach conscious awareness to influence the psychological state of an individual (Craig, 2009). In this context, Mehling et al. (2012) have developed a multidimensional model of the interoceptive construct, which consists of four key dimensions. Firstly, perceptions note subtle changes in body processes indicative of variation in the person's emotional/ physiological state. Secondly, the quality of attention focuses on the emotional reaction and attentional responses to sensations, without being distracted or worried, and includes the subdimension of attentional control. The third includes trust, which reflects the extent to which the individual views awareness of bodily sensations as helpful for decisionmaking or health. Finally, mind-body integration includes emotional awareness, self-regulation, and body listening. Although further research is needed to better understand how interoception functions (Mehling et al., 2018), it is well-known that the quality of internal sensations regulates stress adaptation (Schulz \& Vogele, 2015) and emotional state (Price \& Hooven, 2018). Furthermore, interoceptive efficiency has been described as a particular kind of mindful, nonjudgmental awareness and a sense of self, grounded in physical, external, and internal sensations in the present moment (Mehling et al., 2012). One of the preventive challenges is to identify individual psychological resources that are efficient for dealing with the constraints due to containment. Literature suggests that a pertinent candidate for coping with extreme environments, such as a submarine patrol, could be dispositional mindfulness (Gibson, 2019).

Dispositional mindfulness characterizes the awareness that emerges through paying nonjudgmental, focused attention, in the present moment to the unfolding experience (Kabat-Zinn, 1994). It has been conceptualized as the ability to be consistently mindful in everyday life, regardless of events (Kilpatrick et al., 2011). It is thought to consist of at least two dimensions: acceptance and presence 
(Kabat-Zinn, 1994; Walach et al., 2006). Here, acceptance does not mean resignation, but rather a perception of the experience that simply acknowledges it, rather than judging it as good or bad. Presence is the feeling of being there; it refers to the degree to which a subject is grounded in their own awareness. Dispositional mindfulness is associated with various positive physical and psychological health factors (Brown \& Ryan, 2003), such as efficient emotional and stress regulation (Chiesa \& Serretti, 2009). One potential mechanism for the health benefits associated with mindfulness is a high level of interoceptive sensibility, which can be measured by the Multidimensional Assessment of Interoceptive Awareness (MAIA) questionnaire (Bornemann et al., 2015; Hanley et al., 2017; Mehling et al., 2012). However, evidence of an association between mindfulness and interoception accuracy (operationalized by the heartbeat tracking paradigm) is mixed (Khalsa et al., 2020), and the relationship remains insufficiently characterized. Interestingly, evidence of an association between mindfulness and health behaviors (e.g., eating, sleep quality, and physical activity) is more robust (Gilbert \& Waltz, 2010; Lentz \& Brown, 2019; Roberts \& Danoff-Burg, 2010). On the one hand, dispositional mindfulness appears to be related to decreased stress, which, in turn, contributes to increasingly positive health perceptions and behaviors (Roberts et al., 2010). On the other hand, gender differences have been found with respect to the mindfulness processes that support health behaviors (Gilbert \& Waltz, 2010). Exactly how dispositional mindfulness impacts health behaviors remains a subject of debate. Both emotional (Lentz \& Brown, 2019; Shapiro et al., 2006) and cognitive (Gilbert \& Waltz, 2010) processes have been proposed. However, meta-cognitive processes may also play a part. Intention is one, well-known process that promotes the alignment of daily behavior with personal values and a long-term vision of health, especially in a context of daily stress (Lentz \& Brown, 2019; Shapiro et al., 2006). Concerning sleep, a previous study on SSBN suggested a relationship between subjective and objective sleep assessments without sleep degradation over the patrol (Trousselard et al., 2015). But differences between submariners according to their adaptability were not studied. Referring to the relationship between quality of sleep and dispositional mindfulness in a civilian population (Howell et al., 2008, 2010), it is relevant to evaluate how dispositional mindfulness and sleep are related between each other over the mission using a well-validated questionnaire for a follow-up evaluation of sleep changes as the Leeds Sleep Evaluation Questionnaire (Parrot \& Hindmarch, 1980). Furthermore, higher levels of dispositional mindfulness have been associated with greater awareness of healthy dietary practices and lower tendency to consume food in response to adverse emotional experiences (Lentz \& Brown, 2019). These latter findings suggest that mindfulness may promote health behaviors via interoceptive processes (Hanley et al., 2017). Adjusted assessments for evaluating food attitudes and physical activities, their changes over the patrol, and their relation to interoception functioning are needed for a better understanding of how a patrol impacts these health behaviors.

There are many articles on mindfulness programs to help military personnel manage stress and health during mission, but few articles focus on the impact of mindfulness readiness as a factor in mission adaptation. Furthermore, these articles focus primarily on soldiers, less on Navy personnel, and even less on submariners. To our knowledge, only a few studies have evaluated the psychological benefits of dispositional mindfulness during an SSBN patrol. Our main objective, therefore, is to evaluate the relationship between dispositional mindfulness and health during a SSBN patrol. Our first hypothesis is that dispositional mindfulness remains stable throughout the patrol, despite the artificial environment. The second is that greater dispositional mindfulness is associated with both a better emotional state and better interoception at all stages of the patrol. Third, we hypothesize that dispositional mindfulness is associated with healthier behaviors in terms of diet, sleeping, and exercise. The aim of our work is to explore these points in order to develop individual countermeasures for dealing with ICE missions and/or other confined situations.

\section{Methods}

\section{Participants}

Our exploratory, pragmatic study followed a cohort of male submariners who are declared fit for submarine navigation under French Defense Health Service regulations. This group regularly exercises, as daily sport is an obligatory activity during mission preparations. They are assigned to the French SSBN Le Triomphant, during a patrol in 2018. Although the precise duration is classified, in general, patrols last a minimum of 60 days and a maximum of 80 days. The study was approved by the Comité de Protection des Personnes Sud-Est VI (France), in September 2017 (IDRCB: 2017-A01329-44). Participants were presented with a complete description of this low-risk study, and written informed consent was obtained.

Twenty-four submariners, all volunteers, aged 29.8 years on average ( $\mathrm{SD}=6.45$ ) are included. Twenty-three were in a relationship (95.83\%) and 14 had at least one child (58.33\%). Mean weight was $75.96 \mathrm{~kg}(60-97 \mathrm{~kg}, \mathrm{SD}=9.57)$ and $20(83.33 \%)$ were nonsmokers. Average length of service was $5567 \mathrm{~h}$ on nuclear attack submarines $(\mathrm{SD}=4708)$ and $6835 \mathrm{~h}$ on SSBN (SD =6505). Eight $(33.33 \%)$ were off-watch. This group only worked day shift (no night shifts except for unscheduled interventions). This involved 
a night watch (alternately between 20:00 and 00:00, then 00:00 and 04:00), then between 04:00 and 08:00 on the third night (Trousselard et al., 2015). Shift information was not recorded.

\section{Procedure}

Given that the exact duration of the patrol was unknown, assessments during the mission were scheduled over a period of 60 days. Following consultation with the commander and medical staff, and due to operational constraints, psychological data (dispositional mindfulness and interoception) were collected in four sessions: the first before the patrol (baseline), two during the patrol, on day 25 (D25) and day 55 (D55), and the last, after a month of post-patrol vacation (recovery). Health behaviors (sleep and food behaviors) were only evaluated twice (baseline and recovery). Physical activity was self-reported on a daily basis. All data were collected via self-administered questionnaires. At baseline and recovery, implementation was supported by the SSBN's medical staff and researchers, while at D25 and D50, the SSBN's medical staff worked alone.

\section{Measures}

Sociodemographic data included gender and marital status. The 14-item, self-administered Freiburg Mindfulness Inventory (FMI) assessed mindfulness (Trousselard et al., 2010; Walach et al., 2006). The FMI indexes trait mindfulness as presence and nonjudgmental acceptance. Interoceptive awareness was evaluated using the 32-item MAIA (Mehling et al., 2012), which measures eight facets: (i) noticing (awareness of body sensations), (ii) not-distracting (tendency to distract oneself from negative sensations), (iii) not-worrying (tendency to not worry about negative sensations), (iv) attention regulation (attention to body sensations), (v) emotional awareness (awareness of the connection between body sensations and emotional states), (vi) self-regulation (ability to regulate psychological distress by attention to body sensations), (vii) body listening (actively listening to the body for insight), and (viii) trusting (experience of one's body as safe and trustworthy). Psychological functioning was assessed using the 12-item Scale of Positive and Negative Experience (SPANE), based on how frequently such experiences were felt over the previous 4 weeks (Martin-Krumm et al., 2017).

Variables related to health behaviors during the patrol were recorded as follows. The Leeds Sleep Evaluation Questionnaire (LSEQ) uses ten, 100-mm-line analogue questions (Parrott \& Hindmarch, 1980) to assess four subjective aspects of sleep: getting to sleep, quality of sleep, awakening from sleep, and behavior after waking. The LSEQ was used to monitor subjective changes in sleep with respect to usual subjective sleep. Subjects were asked to indicate current sleep quality compared to their usual sleep. No objective measures of hours slept were recorded. A bespoke, fivepoint Likert scale questionnaire was developed to assess five behaviors toward food and appetite: (i) appetite (little hunger to very hungry); (ii) enjoyment of food (tastes good/bad); (iii) hungry before a meal (never, rarely, sometimes, often or always); (iv) satiety (after a few bites, after one-third or half of the meal, at the end of the meal, still hungry at the end of the meal); and (v) hunger between meals (never, rarely, sometimes, often or always). As meals are served in two courses, at $11 \mathrm{am}$ and $12 \mathrm{pm}$ and then $7 \mathrm{pm}$ and $8 \mathrm{pm}$ with adaptation according to the mission, no objective measures of hours for food consumed were recorded. Daily physical activity was self-reported and divided into three periods during the patrol: the first 20 days, the middle 20 days, and the next 20 days (which are usually the last). Submariners completed a form that indicated the type of activity (strength training, running machine, and/or ergocycle) and the duration of each activity.

\section{Data Analyses}

Data analyses were performed using python software (Python Software Foundation, Wilmington, v3.8) and Statistica (Stastsoft France, Maison Alfort, v7.1). First, the reliability of each psychological measurement (selfadministered questionnaire) was gauged by computing Cronbach's alpha (Cronbach, 1951). All were above 0.74, which indicates good reliability. For FMI, Cronbach's alpha test was 0.78 . Normal distribution and homogeneity of variance were also assessed for quantitative variables using Levene's test. Second, we identified dispositional mindfulness group. A principal component analysis was run to detect relationships between mindfulness subfactors (i.e., presence and acceptance) and to reduce them to a robust, one-dimensional axis. The exploratory factor analysis supported a single-factor solution, which explained $84.08 \%$ of the variance. All variables had a positive weight for this factor-subjects with high scores scored high on mindfulness, while those with low scores scored low on mindfulness. Factor scores were then used to categorize subjects into high and low dispositional mindfulnessgroups using a $k$-means clustering method. Two clusters were identified: the mindful group (MG) consisted of 13 submariners; and the not-mindful group (NMG) consisted of 11 submariners. Third, comparisons between groups were performed using Pearson's chi-square tests for each of the variables with several modalities and $t$ tests for each of quantitative variables. Nonparametric Kruskal-Wallis analyses were used if quantitative data were not normally distributed. Fourth, inter-group comparisons during the patrol were carried out using repeated measures analysis of variances (ANOVAs). 
A first repeated ANOVA was performed for evaluating the stability of dispositional mindfulness during the study with four time-points (Baseline, D25, D55, and Recovery). We, then, examined the effects of time (Baseline, D25, D55, and Recovery) and mindfulness status using (group $\times$ time) ANOVAs for MAIA and SPANE scales, separately. Finally, we examined the effects of time (baseline and recovery) and mindfulness status using (group $\times$ time) ANOVAs for sleep and eating assessments. For physical activity, time (D1-D20, D21-D40, and D41-D60) and mindfulness status (group $\times$ time) effects were examined. When the ANOVA revealed a significant effect, post-hoc Tukey's tests were run for all pairs. Statistical significance was set at $p<0.05$. $p<0.10$ was considered evidence of a trend.

\section{Results}

\section{Mindfulness Status}

No difference was observed between the MG and the NMG with respect to sociodemographic data at baseline. None of the subjects had any experience of meditation. The two groups differed at baseline on the overall FMI score $(\mathrm{F}=56.24 ; p<0.001)$, the presence sub-score $(\mathrm{F}=21.9$; $p<0.001)$, and the acceptance sub-score $(\mathrm{F}=43.96$; $p<0.001)$. The Stability of self-reported mindfulness is described Table 1. A significant group effect $(F=33.9$, $\mathrm{p}<0.001$ ) was observed for FMI scores, with higher scores in the MG. A trend was identified for the interaction between group and time $(\mathrm{F}=2.6, \mathrm{p}=0.06)$ at baseline ( $\mathrm{p}<0.001)$, D25 ( $<<0.001)$, and D55 ( $<<0.001)$. In all three cases, mindfulness scores were lower for NMG, while no difference was found at recovery. Significant group $(F=22.84, p<0.001)$ and time $(F=4.18, p=0.01)$ effects were observed the presence sub-score (Fig. 1, left). Presence scores were higher for the MG. At recovery, presence scores were lower than baseline $(p=0.01)$ and tended to be lower than D25 $(p=0.08)$ and D55 $(p=0.09)$. The interaction identified inter-group differences at baseline $(p=0.01)$, D25 $(p<0.001)$, and D55 $(p=0.002)$ but not recovery $(p=0.83)$. Furthermore, presence scores were lower for the MG group at recovery compared to baseline $(p=0.013)$, D25 $(p<0.001)$, and D55 $(p=0.015)$, while no change was observed for the NMG. A significant group effect $(F=25.84$, $p<0.001)$ highlighted higher scores for the MG at all four times for the acceptance sub-score (Fig. 1, right).

\section{Mindfulness Status and Psychological Adaptation During the Patrol}

Results for SPANE scores differed as a function of the valence of the emotional experience (positive or negative;
Table 1). Scores for positive experiences were higher among the MG than the NMG $(F=10.58, p<0.01)$. Lowest scores were recorded at D55 $(F=15.86, p<0.001)$ and D25 $(p<0.001)$ compared to baseline. There was no group $\times$ time interaction. Turning to negative experiences, an effect of time was observed $(F=3.99, p=0.0151)$, with more negative experiences at D25 $(p=0.02)$ and trends at D55 $(p=0.056)$, and recovery $(p=0.056)$, compared to baseline. The MG tended to experience less negative emotion $(\mathrm{F}=3.03, p=0.10)$. An inter-group trend was identified, but no interaction.

Significant group $(F=12.18, p=0.003)$ and time $(F=4.48, p=0.007)$ effects were found for MAIA scores (Table 1). They highlighted that the MG scored higher for interoception, and that interoception decreased as the patrol progressed. Scores were highest at baseline and D25, compared to D55 $(p=0.01)$ and recovery $(p=0.01)$. Significant group and time effects differed according to the eight MAIA sub-scores. No effect was observed for noticing. Scores for not-distracting $(F=4.3, p=0.008)$ were higher at baseline compared to D55 $(p=0.027)$ and recovery $(p=0.028)$. Scores for not-worrying $(F=6.87, p<0.001)$ were higher at baseline compared to D25 $(p<0.001)$, D55 $(p=0.042)$, and recovery $(p=0.005)$. Scores for attention regulation $(F=19.9, p<0.001)$ were higher among the MG than the NMG. A time trend $(F=2.4, p=0.08)$ suggests that attention regulation was higher at baseline than D55 $(p=0.04)$. A group trend was identified for emotional awareness $(F=3.3$, $p=0.09$ ) with higher scores for the MG compared to the NMG. A group effect was identified for self-regulation $(F=17, p<0.001)$, body listening $(F=9.22, p=0.008)$, and trusting $(F=5.04, p=0.04)$, with the MG scoring higher than the NMG in all cases.

Table 2 presents submariners' scores for sleeping, eating, and physical activity assessments as a function of mindful status. The only notable result for sleep was a trend for waking at night $(F=0.396 ; p=0.06)$. The $\mathrm{MG}$ tended to experience less waking at baseline and recovery compared to the NMG. For getting to sleep scales, a time effect was observed for sleep latency $(F=7.46 ; p=0.014)$ and feeling sleepy $(F=7.72 ; p=0.013)$. At recovery, latency returns to normal, while greater difficulty is reported in falling asleep (compared to usual) at baseline. The same result is observed for feeling sleepy. A time effect is observed for restless/restful sleep $(F=5.24 ; p=0.035)$. Sleep is reported to be more restful at recovery than baseline. For waking during sleep, time effects were observed. Scores for waking up easily tended to be better at baseline than recovery $(F=3.32 ; \mathrm{p}=0.08)$. In the same vein, waking up was slower at recovery compared to baseline $(F=8.65 ; p=0.01)$. Postwakening, fatigue, balance, and coordination tended to differ between baseline and recovery. Less fatigue than usual was reported at baseline, while at recovery, it was the same as 


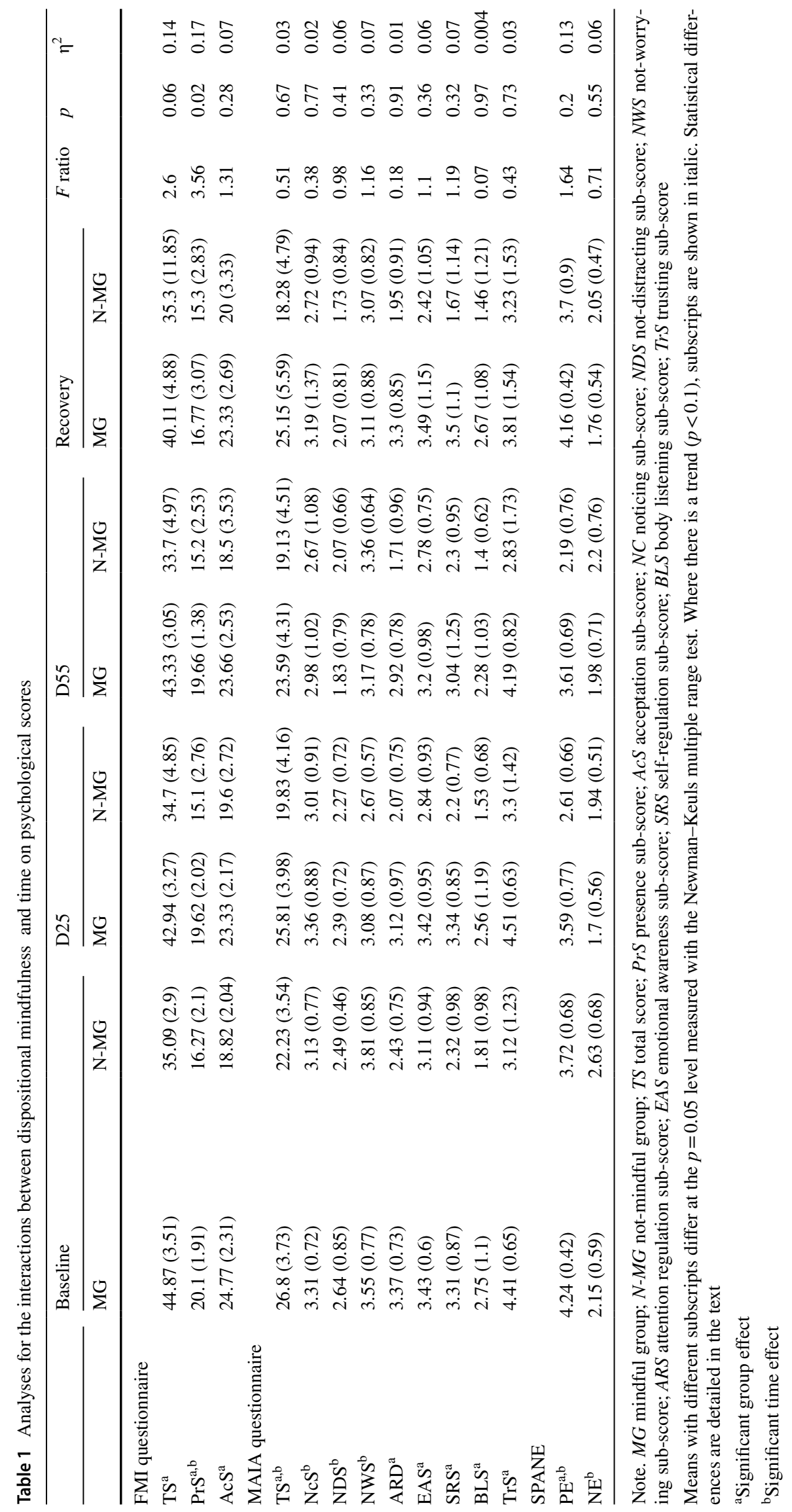



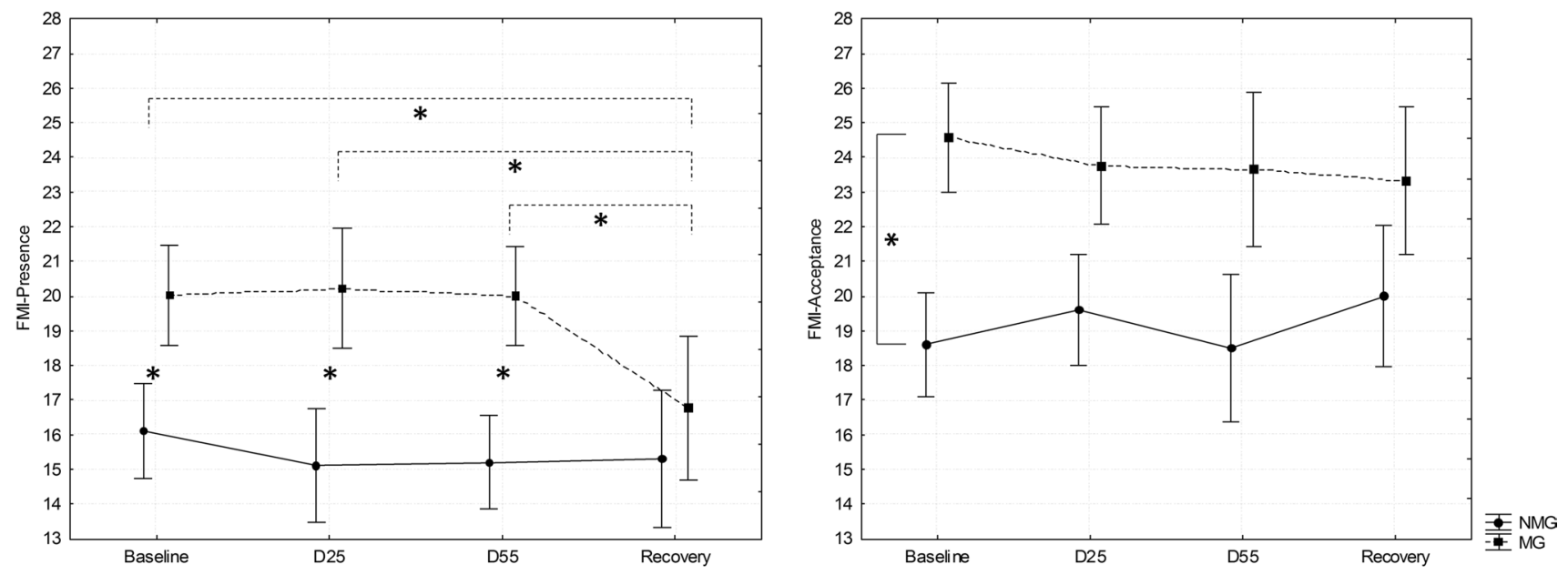

Fig. 1 Significant group (NMG and MG) and time (baseline, D25, D55, and recovery) interactions for FMI presence (left) and FMI acceptance sub-scores. Solid line*, inter-group difference; dotted line*, intersession difference (MG only)

usual $(F=3.96 ; p=0.06)$. Balance and coordination were reported to be less perturbed than usual at baseline, but the same as usual at recovery $(F=4.27 ; p=0.054)$. Hunger before and hunger between meals were lower at recovery compared to baseline $(F=8.77 ; p=0.009$ and $F=18.04$; $p<0.001$, respectively). A significant interaction between group and hunger before meals revealed that the MG was less hungry at recovery compared to baseline $(p=0.004)$, while no change was observed for the NMG $(p=0.66)$. Turning to physical activity during the patrol, the mean for the MG was $22.61(\mathrm{SD}=18.91)$ days of activity and a total of $1502.3(\mathrm{SD}=1847.41) \mathrm{h}$. The mean for the NMG was

Table 2 Analyses of interactions between mindfulness trait and time on health behaviors

\begin{tabular}{|c|c|c|c|c|c|c|c|c|c|}
\hline & & Mindfulness & Baseline $\mathrm{M}(\mathrm{S})$ & & Recovery M(S & D) & $F$ ratio & $p$ & $\eta^{2}$ \\
\hline \multirow{8}{*}{ Sleep } & \multirow{4}{*}{ GTS } & & MG & N-MG & MG & N-MG & & & \\
\hline & & Difficult/easy & $2.84(1.99)$ & $2.81(2.31)$ & $0.33(3.77)$ & $2.3(3.68)$ & 0.82 & 0.37 & 0.05 \\
\hline & & Sleep latency & $2.54(2.02)$ & $2.54(2.46)$ & $-0.66(3.9)$ & $1.1(4.09)$ & 0.91 & 0.35 & 0.05 \\
\hline & & Sleepiness & $1.38(3.28)$ & $2.36(1.29)$ & $-0.66(3)$ & $1(3.12)$ & 1.11 & 0.30 & 0.06 \\
\hline & \multirow[t]{2}{*}{ QoS } & Restless/restful ${ }^{\text {b }}$ & $-2.69(2.43)$ & $-1.72(2.41)$ & $-3.33(1)$ & $-3.3(1.77)$ & 0.51 & 0.48 & 0.02 \\
\hline & & Wakefulness ${ }^{b}$ & $-0.55(3.47)$ & $0(2.65)$ & $-0.55(2.92)$ & $0.5(3.5)$ & 0.08 & 0.77 & $<0.01$ \\
\hline & \multirow[t]{2}{*}{ AFS } & Difficult/easy & $1.84(2.88)$ & $1.27(3.16)$ & $0.22(3.19)$ & $0.5(3.21)$ & 0.47 & 0.49 & 0.03 \\
\hline & & Slow/quick & $2.23(2.35)$ & $1.9(2.94)$ & $-1.11(3.16)$ & $0.3(2.67)$ & 1.63 & 0.22 & 0.09 \\
\hline \multirow{8}{*}{$\begin{array}{l}\text { Food } \\
\text { attitudes }\end{array}$} & \multirow[t]{3}{*}{ BFW } & Fatigue when wake up & $2.69(2.05)$ & $1.18(2.13)$ & $-0.77(3.63)$ & $0.8(2.69)$ & 2.82 & 0.11 & 0.14 \\
\hline & & Tiredness now & $3.23(1.01)$ & $1.36(2.37)$ & $-0.33(4.15)$ & $1.8(2.78)$ & 5.55 & 0.03 & 0.24 \\
\hline & & $\begin{array}{l}\text { Balance and coordina- } \\
\text { tion }\end{array}$ & $3.23(1.79)$ & $2.45(2.42)$ & $0.22(4.57)$ & $1(3.62)$ & 0.61 & 0.44 & 0.03 \\
\hline & \multicolumn{2}{|l|}{ Appetite } & $4.15(0.89)$ & $3.63(0.81)$ & $4.11(0.6)$ & $3.9(0.57)$ & 1.23 & 0.28 & 0.07 \\
\hline & \multicolumn{2}{|l|}{ Enjoyment of food } & $4.31(0.48)$ & $3.9(0.54)$ & $4.11(0.6)$ & $4(0.47)$ & 0.89 & 0.35 & 0.05 \\
\hline & \multicolumn{2}{|l|}{ Hunger when time to eat ${ }^{b}$} & $4.08(0.86)$ & $3.72(0.78)$ & $3.34(0.53)$ & $3.7(0.48)$ & 8.76 & 0.001 & 0.34 \\
\hline & \multicolumn{2}{|l|}{ Satiety after eating } & $4.07(0.49)$ & $3.91(0.53)$ & $4(0.1)$ & $4.1(0.31)$ & 1.2 & 0.28 & 0.06 \\
\hline & \multicolumn{2}{|l|}{ Hunger between meals ${ }^{b}$} & $3.15(0.90)$ & $3.09(0.7)$ & $2.33(0.5)$ & $2.3(0.82)$ & 1.49 & 0.70 & 0.008 \\
\hline
\end{tabular}

Note. $M G$ mindful group; $N-M G$ not-mindful group; $G T S$ getting to sleep; $Q o S$ quality of sleep; $A F S$ awakening from sleep; $B F W$ behavior following wakefulness

Means with different subscripts differ at the $p=0.05$ level measured with the Newman-Keuls multiple range test. Where there is a trend $(p<0.10)$, subscripts are shown in italic

${ }^{\text {a }}$ Significant group effect

${ }^{\mathrm{b}}$ Significant time effect 
$16.82(\mathrm{SD}=16.03)$ days of practice and a total of 970.1 $(\mathrm{SD}=1450.92)$ h. No significant difference was observed between the two groups. Physical activity during the patrol (number of days) also did not differ between the two groups for the three periods. A slight interaction was observed between group and period $(F=2.67 ; p=0.08)$. At the end of the patrol (D41-D60), the number of days of practice tended to be higher compared to the first period (D1-D20) for the MG, while it did not change for the NMG.

\section{Discussion}

The aim of this study was to evaluate whether dispositional mindfulness is a resource for coping with a professional environment that is characterized by long-term containment. The observed decrease in positive emotion, along with an increase in negative emotion as the patrol progressed, confirms that the SSBN is a stressful environment that challenges human adaptation. First, we tested whether self-reported measures of mindfulness disposition were stable across the four experimental sessions (including during the patrol). To the best of our knowledge, no study has investigated dispositional mindfulness as a psychological resource, while it is thought to be relatively stable over time, even in stressful situations. Our findings confirm that dispositional mindfulness was stable during the patrol, especially the acceptance dimension. Interestingly, the presence dimension tended to decrease for the MG, but not the NMG. Body self-awareness can be broadly defined as the extent to which people are consciously aware of their bodily states and their relationships with others (Sutton, 2016). This conscious feeling of a unitary entity is shaped by exteroceptive and interoceptive signals, and maintained by the integration of sensory signals (Blanke, 2012). During the patrol, interoception scores were higher for the MG, but decreased from the middle of the patrol. This suggests that subjects with high dispositional mindfulness, but no experience of meditation, modify the connection between their body and the environment during immersion in an artificial situation (such as a submarine patrol). However, although presence scores fell in the MG, their emotional state during the patrol remained better than the NMG, confirming our second hypothesis. Furthermore, the impact of the patrol differed according to the interoceptive dimension and mindful status. Overall, during the patrol, scores fell (or tended to fall) for not-distracting, not-worrying, and attention regulation. At the same time, the MG scored higher than the NMG for attention regulation, self-regulation, emotional awareness, body listening, and trusting. With the exception of attention regulation, these differences during the patrol depended on either the time or mindful status. These changes are in line with theories of interoception (Garfinkel et al., 2015; Mehling et al., 2012). We adopt Mehling's concept of interoception, which sees it as a multidimensional construct with four key dimensions (Mehling et al., 2012, 2018). Our results suggest that the patrol per se impacts the first dimension (perceived body sensations), while dispositional mindfulness interacts with integrative dimensions. Attention regulation (which is presented as an intermediate step between perceived body sensations and integrative dimensions) should, therefore, be pivotal as it integrates the effects of the environment and personal interoceptive abilities. It could indicate the integration of internal and external perceptions of the body, which, in turn, contributes to the regulation of physiological integrity (homeostasis) and associated affect, drives, and emotions. Turning to our third hypothesis, although the patrol is associated with changes in health behavior, dispositional mindfulness had little impact on these changes. Self-reported sleep was poorer before the patrol (falling asleep, quality of sleep, awakening during sleep, and waking up) than at recovery. Nevertheless, the MG tended to report fewer waking periods at baseline and recovery compared to the NMG. Self-reported eating habits indicated that recovery was associated with less hunger at meal times and between meals, especially for the MG. Finally, physical exercise patterns did not change significantly during the patrol, although the MG tended to exercise a little more at the end. No data are available from during the patrol, and it is possible that health behaviors could have been disturbed at the very start, but returned to their usual level at recovery.

Furthermore, the impacts of the patrol can be understood as resulting from the SSBN environment. The latter confines individuals in a context that is characterized by a lack of sensory stimulation, a lack of variation in stimulation, or overstimulation. A healthy subject, who is living and working in a non-ecological environment, such as Antarctic and space bases, may integrate interoceptive signals differently compared to the usual ecological environment (Rivolier, 1992). Although Antarctic bases, space bases, and submarines are considered analogous, Antarctic bases are an unusual and extreme, but natural environment, while space bases and submarines are unusual and extreme artificial environments. The impact of this difference needs to be further evaluated in human adaptation studies. This observation may have applications for the current COVID-19 quarantine period, which decreases sensory stimulation from the environment. It also questions the interaction between interoceptive and exteroceptive cues, although it is not clear whether a supramodal form of bodily self-awareness exists (Salvato et al., 2020). In addition, for individuals who are confined in an environment with little exteroceptive stimulation, dispositional mindfulness may not be enough to maintain the ability to be fully conscious and aware in the present moment, which could explain the decrease in dispositional mindfulness during our experiment. Altogether, our results suggest that two countermeasures could be developed for dealing with ICE (Fig. 2). The first would be to use sensory stimulation to 
Fig. 2 Relationship between interoception models and impacts of the mission for submariners

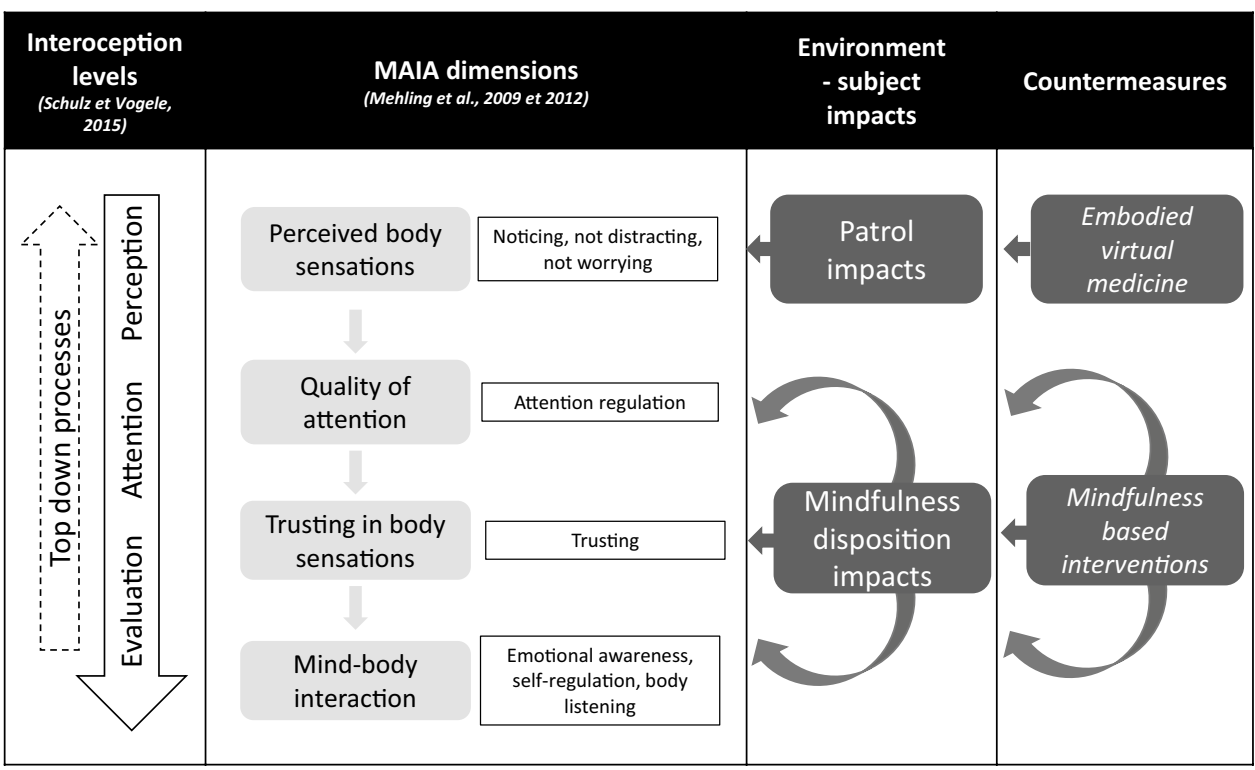

enhance both exteroceptive and interoceptive information. It appears to be advisable to increase natural sensory stimuli if possible (Gould van Praag et al., 2017; Sarris et al., 2019). As a complement, or if this is not possible, countermeasures could include virtual reality and computer-based virtual environments. A multisensory platform could modulate both external and internal bodily information, and improve bodily self-awareness (Duquette, 2017). This emerging approach is the subject of a new, transdisciplinary research fieldembodied medicine (Riva et al., 2017)—which aims to improve health and well-being using advanced technology to alter multisensory representations. A second countermeasure is mindfulness meditation. Over the past three decades, research has paid much more attention to its benefits for cognitive functioning (Sedlmeier et al., 2012), health and well-being (for a review, see $\mathrm{Gu}$ et al., 2015), and, more recently, interoceptive abilities (Fissler et al., 2016). Mindfulness meditation could be helpful in dealing with containment, even for subjects with a high level of dispositional mindfulness (Gibson, 2019).

\section{Limitations and Future Research Direction}

The present study has important limitations. First, from a clinical point a view, to our knowledge, there is no literature on evaluating the clinical relevance of the difference observed between groups on FMI tool. Cluster analysis based on FMI scores revealed two groups with an average difference of two points between them. The observed statistical differences need to be further evaluated on their clinical relevance. Second, there are several limitations to our exploratory study and several avenues for further research. The first limitation concerns the studied population. The sample was small, and male. The second comes from the use of self-reported measures, as there are limitations inherent in the approach (e.g., response bias, state dependencies, and social desirability). In line, because of the submariners' duty of reserve, it makes difficult to access the quality of cohesion due to food sharing during the mission. An evaluation of the relation between food attitudes and cohesion would be relevant in further studies. Furthermore, the repeated administration of the interoceptive scale revealed low internal consistency for not-distracting and not-worrying dimensions (Mehling et al., 2018). As the patrol impacted these two scales, the recent addition of three items to each of them needs to be included in further studies in order to confirm (or not) our results. Finally, the health behaviors were only selfreported during the patrol. Objective measures using monitoring for sleep, sport, or food intake would be necessary for better assessing how health behaviors change inside the submarine during the patrol. This will be helpful for evaluating the clinical relevance of the subjective changes that we have recorded during the mission. Future work could address these limitations, and this would provide a better understanding of how mindfulness can be used as a preventive measure in both isolated and confined environments, and quarantine.

Acknowledgements The authors wish to thank the officer directing the SSBN for their help throughout the process. We thank the submariners for their involvement.

Author Contribution CA-P, AV, and MT conceived the study. MT and $\mathrm{FC}$ wrote the protocol. All authors actively took part in the process. All authors have planned and participated in the statistical analysis. AL, JP, FL, LG, and AV recorded data over the patrol. AD, CMK, FR, and $\mathrm{CR}$ recorded data before and at recovery. $\mathrm{MT}, \mathrm{LG}$, and $\mathrm{CMK}$ wrote the paper. JBB, FC, and FD corrected all the text and designed the figures. All authors read and approved the final manuscript. 


\section{Declarations}

Ethics Approval The study was approved by the "Comité de Protection des Personnes Sud-Est VI (France)" on September 2017 (IDRCB: 2017-A01329-44).

Consent to Participate After a complete description of the study, a written informed consent was individually obtained for each voluntary for participation using the validated information and consent letters by the committee.

Conflict of Interest The authors declare no competing interests.

\section{References}

Blanke, O. (2012). Multisensory brain mechanisms of bodily self-consciousness. Nature Reviews Neuroscience, 13, 556-571. https:// doi.org/10.1038/nrn3292

Bornemann, B., Herbert, B. M., Mehling, W. E., \& Singer, T. (2015). Differential changes in self-reported aspects of interoceptive awareness through 3 months of contemplative training. Frontiers in Psychology, 5, 1504. https://doi.org/10.3389/fpsyg.2014.01504

Bouillon-Minois, J.-B., Trousselard, M., \& Dutheil, F. (2020). Covid19 pandemic containment: Following the example of military submariners. BMJ Military Health, 0, 1. https://doi.org/10.1136/ bmjmilitary-2020-001501.

Brasher, K. S., Dew, A. B. C., Kilminster, S. G., \& Bridger, R. S. (2010). Occupational stress in submariners: The impact of isolated and confined work on psychological well-being. Ergonomics, 53(3), 305-313. https://doi.org/10.1080/00140130903067763

Brown, K. W., \& Ryan, R. M. (2003). The benefits of being present: Mindfulness and its role in psychological well-being. Journal of Personality and Social Psychology, 84(4), 822-848. https://doi. org/10.1037/0022-3514.84.4.822

Chiesa, A., \& Serretti, A. (2009). Mindfulness-based stress reduction for stress management in healthy people: A review and metaanalysis. Journal of Alternative and Complementary Medicine, 15(5), 593-600. https://doi.org/10.1089/acm.2008.0495

Craig, A. D. (2009). How do you feel now? The anterior insula and human awareness. Nature Reviews Neuroscience, 10(1), 59-70. https://doi.org/10.1038/nrn2555

Cronbach, L. J. (1951). Coefficient alpha and the internal structure of tests. Psychometrika, 16, 297-334.

Duquette, P. (2017). Increasing our insular world view: Interoception and psychopathology for psychotherapists. Frontiers in Neuroscience, 11, 135. https://doi.org/10.3389/fnins.2017.00135

Eid, J., Johnson, B. H., \& Thayer, J. F. (2001). Post-traumatic stress symptoms following shipwreck of a Norwegian Navy frigate An early follow-up. Personality and Individual Differences, 30 , 1283-1295.

Fissler, M., Winnebeck, E., Schroeter, T., Gummersbach, M., Huntenburg, J. M., Gaertner, M., \& Barnhofer, T. (2016). An investigation of the effects of brief mindfulness training on self-reported interoceptive awareness, the ability to decenter, and their role in the reduction of depressive symptoms. Mindfulness, 7, 1170 1181. https://doi.org/10.1007/s12671-016-0559-z

Garfinkel, S. N., Seth, A. K., Barrett, A. B., Suzuki, K., \& Critchley, H. D. (2015). Knowing your own heart: Distinguishing interoceptive accuracy from interoceptive awareness. Biological Psychology, 104, 65-74. https://doi.org/10.1016/j.biopsycho.2014.11.004

Gilbert, D., \& Waltz, J. (2010). Mindfulness and health behaviors. Mindfulness, 1, 227-234. https://doi.org/10.1007/ s12671-010-0032-3
Gibson, J. (2019). Mindfulness, interoception, and the body: A contemporary perspective. Frontiers in Psychology, 10, 2012. https:// doi.org/10.3389/fpsyg.2019.02012

Gould van Praag, C., Garfinkel, S., Sparasci, O., Mess, A., Philippides, A. O., Ware, M., Ottaviani, C., \& Critchley, H. D. (2017). Mindwandering and alterations to default mode network connectivity when listening to naturalistic versus artificial sounds. Sciences Reports, 7, 45273. https://doi.org/10.1038/srep45273

Gu, J., Strauss, C., Bond, R., \& Cavanagh, K. (2015). How do mindfulness-based cognitive therapy and mindfulness-based stress reduction improve mental health and wellbeing? A systematic review and meta-analysis of mediation studies. Clinical Psychology Review, 37, 1-12. https://doi.org/10.1016/j.cpr.2015.01.006

Hanley, A. W., Mehling, W. E., \& Garland, E. (2017). Holding the body in mind: Interoceptive awareness, dispositional mindfulness and psychological well-being. Journal of Psychosomatic Research, 99 , 13-20. https://doi.org/10.1016/j.jpsychores.2017.05.014

Howell, A. J., Digdon, N. L., Buro, K., \& Sheptycki, A. R. (2008). Relations among mindfulness, well-being, and sleep. Personality and Individual Differences, 45, 773-777. https://doi.org/10. 1016/j.paid.2008.08.005

Howell, A. J., Digdon, N. L., \& Buro, K. (2010). Mindfulness predicts sleep-related self-regulation and well-being. Personality and Individual Differences, 48(4), 419-424. https://doi.org/10.1016/j. paid.2009.11.009

Kabat-Zinn, J. (1994). Wherever you go, there you are: Mindfulness meditation in everyday life. Hyperion.

Khalsa, S. S., Rudrauf, D., Hassanpour, M. S., Davidson, R. J., \& Tranel, D. (2020). The practice of meditation is not associated with improved interoceptive awareness of the heartbeat. Psychophysiology, 57(2), e13479. https://doi.org/10.1111/psyp.13479

Kilpatrick, L. A., Suyenobu, B. Y., Smith, S. R., Bueller, J. A., Goodman, T., Creswell, J. D., Tillisch, K., Mayer, E. A., \& Naliboff, B. D. (2011). Impact of mindfulness-based stress reduction training on intrinsic brain connectivity. NeuroImage, 56(1), 290-298. https://doi.org/10.1016/j.neuroimage.2011.02.034

Lentz, T. A., \& Brown, C. (2019). Mindfulness and health behaviors in college students: The moderating role of sleep. Journal of American College Health, 67(6), 505-514. https://doi.org/10. 1080/07448481.2018.1497638

Levit-Binnun, N., \& Golland, Y. (2012). Finding behavioral and network indicators of brain vulnerability. Frontiers in Human Neuroscience, 6, 10. https://doi.org/10.3389/fnhum.2012.00010

Martin-Krumm, C., Fenouillet, F., Csillik, A., Kern, L., Besancon, M., Heutte, J., Paquet, Y., Delas, Y., Trousselard, M., Lecorre, B., \& Diener, E. (2017). Changes in Emotions from Childhood to Young Adulthood. Child Indicator Research, 11(2), 541-561. https://doi. org/10.1007/s12187-016-9440-9

Mehling, W. E., Gopisetty, V., Daubenmier, J., Price, C. J., Hecht, F. M., \& Stewart, A. (2009). Body awareness: Construct and selfreport measures. PLoS ONE, 4(5), e5614. https://doi.org/10.1371/ journal.pone.0005614

Mehling, W. E., Price, C., Daubenmier, J. J., Acree, M., Bartmess, E., \& Stewart, A. (2012). The Multidimensional Assessment of Interoceptive Awareness (MAIA). PLoS ONE, 7(11), e 48230. https://doi.org/10.1371/journal.pone.0048230

Mehling, W. E., Acree, M., Stewart, A., Silas, J., \& Jones, A. (2018). The Multidimensional Assessment of Interoceptive Awareness, Version 2 (MAIA-2). PLoS ONE, 13(12), e0208034. https://doi. org/10.1371/journal.pone.0208034

Palinkas, L. A., Glogower, F. G., Dembert, M., Hansen, K., \& Smullen, R. (2004). Incidence of psychiatric disorders after extended residence in Antarctica. International Journal of Circumpolar Health, 63, 157-168. https://doi.org/10.3402/ijch.v63i2.17702

Palinkas, L. A., Reed, H. L., \& Do, N. V. (1997). Association between the Polar T Syndrome and the Winter-Over Syndrome in 
Antarctica. Antarctic Journal of the United States Review, 32, $112-114$

Palinkas, L. A., Reedy, K., Shepanek, M., Smith, M., Anghel, M., Steel, G. D., Reeves, D., Case, H. S., Do, N. V., \& Reed, H. L. (2007). Environmental influences on hypothalamic-thyroid function and behavior in Antarctica. Physiology and Behavior, 92, 790-799. https://doi.org/10.1016/j.physbeh.2007.06.008

Parrott, A. C., \& Hindmarch, I. (1980). The Leeds sleep evaluation questionnaire in psychopharmacological investigations - A review. Psychopharmacology (berl), 71(2), 173-179. https://doi.org/10. 1007/BF00434408

Price, C. J., \& Hooven, C. (2018). Interoceptive awareness skills for emotion regulation: Theory and approach of mindful awareness in body-oriented therapy (MABT). Frontiers in Psychology, 9, 798. https://doi.org/10.3389/fpsyg.2018.00798

Riva, G., Serino, S., Di Lernia, D., Pavone, E. F., \& Dakanalis, A. (2017). Embodied medicine: Mens sana in corpore virtuale sano. Frontiers in Human Neuroscience, 11, 120. https://doi.org/10. 3389/fnhum.2017.00120

Rivolier, J. (1992). Facteurs humains et situations extremes [Human factors and extreme environment]. Masson; coll. Médecine et psychothérapie.

Roberts, K. C., \& Danoff-Burg, S. (2010). Mindfulness and health behaviors: Is paying attention good for you? Journal of American College Health, 59(3), 165-173. https://doi.org/10.1080/07448 481.2010.484452

Salvato, G., Richter, F., Sedeño, L., Bottini, G., \& Paulesu, E. (2020). Building the bodily self-awareness: Evidence for the convergence between interoceptive and exteroceptive information in a multilevel kernel density analysis study. Human Brain Mapping, 41, 401-418. https://doi.org/10.1002/hbm.24810

Sarris, J., De Manincor, M., Hargraves, F., \& Tsonis, J. (2019). Harnessing the Four Elements for Mental Health. Frontiers in Psychiatry, 10, 256. https://doi.org/10.3389/fpsyt.2019.00256

Sedlmeier, P., Eberth, J., Schwarz, M., Zimmermann, D., Haarig, F., Jaeger, S., \& Kunze, S. (2012). The psychological effects of meditation: A meta-analysis. Psychological Bulletin, 138(6), 1139-1171. https://doi.org/10.1037/a0028168

Schulz, A., \& Vogele, C. (2015). Interoception and stress. Frontiers in Psychology, 6, 993. https://doi.org/10.3389/fpsyg.2015.00993

Shapiro, S., Carlson, L. E., Astin, J. A., \& Freedman, B. (2006). Mechanism of mindfulness. Journal of Clinical Psychology, 62(3), 373-386. https://doi.org/10.1002/jclp.20237

Sutton, A. (2016). Measuring the effects of self-awareness: Construction of the self-awareness outcomes questionnaire. Europe's Journal of Psychology, 12(4), 645-658. https://doi.org/10.5964/ejop. v12i4.1178

Trousselard, M., Steiler, D., Raphel, C., Cian, C. D., \& R, Claverie, D., \& Canini, F. . (2010). Validation of a French version of the Freiburg Mindfulness Inventory-short version: How mindfulness deals with the stress in a working middle-aged population. Biopsychosocial Medicine, 4(8), 1-11. https://doi.org/10.1186/ 1751-0759-4-8

Trousselard, M., Chennaoui, M., Coste, O., Rabat, A., Van Beers, P., \& Leger, D. (2015). Sleeping under ocean. PLOS ONE, 10(5), e0126721. https://doi.org/10.1371/journal.pone.0126721

Vanhove, A., Herian, M., Harms, P., Luthans, F., \& Desimone, J. (2015). Examining psychosocial well-being and performance in isolated, confined, and extreme environments. Report number: NASA/TM-2015-218565.

Walach, H., Buchheld, N., Buttenmüller, V., Kleinknecht, N., \& Schmidt, S. (2006). Measuring mindfulness-the Freiburg Mindfulness Inventory (FMI). Personality and Individual Differences, 40(8), 1543-1555. https://doi.org/10.1016/j.paid.2005.11.025

Publisher's Note Springer Nature remains neutral with regard to jurisdictional claims in published maps and institutional affiliations. 